Derechos reservados de El Colegio de Sonora, ISSN 1870-3925

\title{
Tarifas y consumo de agua en el sector residencial de la Comarca Lagunera
}

\section{J. Alberto García-Salazar* \\ J. Saturnino Mora-Flores*}

Resumen: La escasez de agua en la Comarca Lagunera justifica la elaboración de estudios para analizar la demanda del recurso entre los usuarios. Para determinar los factores que influyen en la cantidad del líquido consumida en la zona metropolitana de la Comarca Lagunera, se estimaron dos funciones de demanda en el sector urbano de Torreón, Coahuila y Gómez Palacio, Durango. Los resultados indican que ésta responde de manera inelástica a cambios en las tarifas, con elasticidades de -0.20 y -0.18 para cada ciudad, respectivamente; que el gasto aumenta conforme se incrementan los ingresos del consumidor, y que el agua es un bien complementario de la energía eléctrica. Debido a la baja elasticidad precio de la demanda, no se recomienda subir las tarifas en el tiempo para disminuir el consumo, pues las alzas tendrían repercusiones reducidas sobre la cantidad usada.

Palabras clave: agua, demanda, elasticidad, tarifas, electricidad, residencial.

Abstract: The scarcity of water in the Comarca Lagunera justifies studies analyzing water demand among consumers. For detecting which factors affect the amount of water used in the metropolitan area of the Comarca Lagunera, two water demand functions in the urban sector of Torreón, Coahuila, and Gómez Palacio, Durango were estimated. The results indicate that the water used responded

\footnotetext{
* Colegio de Postgraduados. Km. 36.5 carretera México-Texcoco. C.P. 56230, Montecillo, Estado de México. Teléfono 01 (595) 95202 00, extensión 1836. Correo electrónico: jsalazar@colpos.mx
} 
in an inelastic way to changes in tariffs, with elasticities of -0.20 and -0.18 for each city, respectively; that water consumption increases as consumer income rises, and that water and electricity are complementary goods. Due to the low price elasticity of water demand, increasing rates over time is not recommended for reducing consumption, because such tariff increases would have little effect on the amount of water used.

Key words: water, demand, elasticity, tariffs, electricity, residential.

\section{Introducción}

La Comarca Lagunera es, sin duda alguna, una de las regiones más prósperas de México. En la producción pecuaria ocupa un lugar relevante en la ganadería de leche, carne de pollo y huevo. La minería está en los primeros sitios en la exportación de oro, plata y minerales no ferrosos, a través de la industria Peñoles. La producción textil es importante debido a que es la principal exportadora nacional de mezclilla.

Está integrada por cinco municipios de Coahuila (Francisco I. Madero, Matamoros, San Pedro, Torreón y Viesca) y diez de Durango (Simón Bolívar, Gómez Palacio, Lerdo, Mapimí, Nazas, Rodeo, San Juan de Guadalupe, San Luis del Cordero, San Pedro del Gallo y Tlahualilo) (véase mapa). Desde el punto de vista demográfico, es uno de los núcleos urbanos más grandes del norte del país, formado por las ciudades de Torreón, Gómez Palacio y Lerdo, que sumaban 828 mil habitantes en 2002 (Secretaría de Agricultura, Ganadería, Desarrollo Rural, Pesca y Alimentación, SAGARPA 2003, 12). Existen también los poblados medios de entre 10 mil y 60 mil habitantes: Matamoros, San Pedro, Francisco I. Madero, Mapimí y Tlahualilo; además otras localidades pequeñas, dispersas en todo el territorio, que junto con las medianas alojan 446 mil personas.

El abastecimiento del líquido para la zona metropolitana de la región está a cargo de tres organismos operadores: el Sistema Municipal de Aguas y Saneamiento (SIMAS), que suministra y administra el recurso en Torreón; el Sistema Descentralizado de Agua Potable y Alcantarillado (SIDEAPA), que abastece a Gómez Palacio y el Sistema de Agua Potable y Alcantarillado (SAPAL), que alimenta a Lerdo.

Para dotar de agua a más de 1.2 millones de habitantes, se necesitan 4.3 $\mathrm{m}^{3}$ por segundo; para lograrlo, los sistemas operadores extraen el recurso de 


\section{Mapa}

Ubicación de la Comarca Lagunera: Torreón, Coahuila y Gómez Palacio, Durango

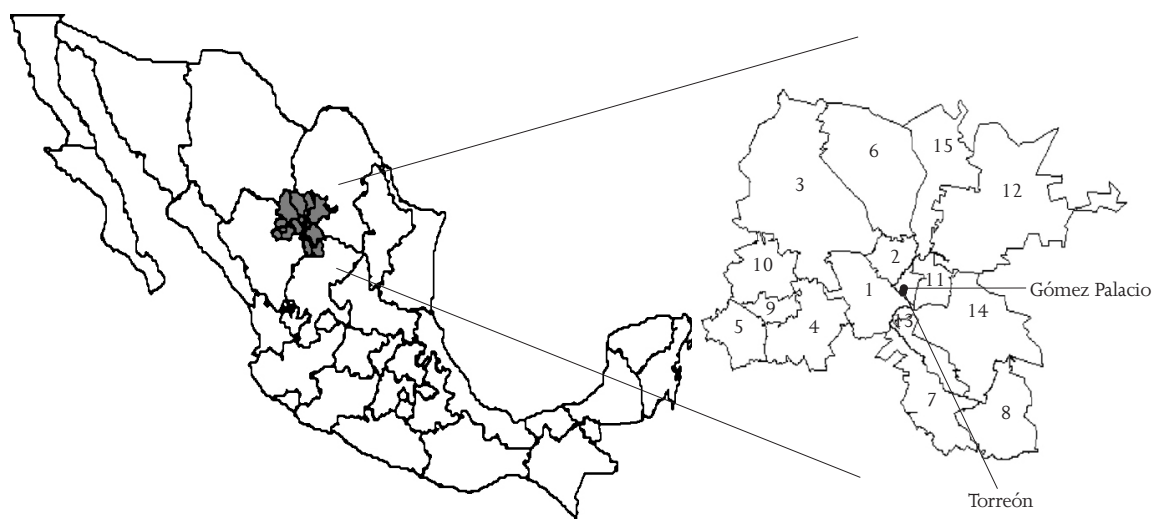

Municipios en Durango

1. Lerdo

2. Gómez Palacio

3. Mapimí

4. Nazas

5. Rodeo

6. Tlahualilo

7. Simón Bolívar

8. San Juan de Guadalupe

9. San Luis del Cordero

10. San Pedro del Gallo
Municipios en Coahuila

11. Matamoros

12. San Pedro

13. Torreón

14. Viesca

15. Francisco I. Madero

la "burbuja”, mayor depósito subterráneo del acuífero Principal, ${ }^{1}$ que está libre de arsénico y otros minerales nocivos para la salud humana.

Torreón se abastece de 60 pozos profundos; 18 en el norte, 18 en el centro y 24 en el sur de la ciudad. Su profundidad es variable; desde 125 hasta 450 metros. La oferta de agua para la ciudad es de más de 2200 litros por segundo, lo cual supera los 70 millones de $\mathrm{m}^{3}$ al año. Tal volumen satisface las necesidades de los usuarios domésticos, industriales, comerciales y de servicios, también incluye las pérdidas en el sistema de distribución. El SIMAS de Torreón tiene registradas más de 130 mil tomas, de las cuales 90.6 por

\footnotetext{
1 La zona geohidrológica de la Comarca Lagunera está conformada por ocho acuíferos: Principal,
} Acatita, Las Delicias, Villa Juárez, Ceballos, Oriente Aguanaval, Nazas y Vicente Suárez. Por la magnitud de las reservas, el Principal es el más importante. 
ciento son domésticas, 9.0 comerciales, 0.3 industriales y 0.02 son para otros usos, como servicios comerciales (Comisión Nacional del Agua, CNA 1997, 112).

El suministro de agua para Gómez Palacio, segunda ciudad en importancia en la región, con más de 200 mil habitantes, se realiza mediante la explotación de 34 pozos profundos, de los que se extraen 848 litros por segundo. Aquí se han definido tres tipos de consumo doméstico promedio: popular, medio y alto, que gastan 185, 230 y 400 litros, respectivamente (CNA 1997, 125).

El sistema de tarifas por rango de consumo se presenta en el cuadro 1; están diferenciadas para cada tipo de gasto, son mayores para cantidades más grandes. El sistema de cobro de Torreón considera siete bloques de consumo e igual número de cuotas; el sistema de Gómez Palacio toma en cuenta once bloques (véase cuadro 1). En Torreón se aplica un solo precio a consumidores de bajos, medios y altos ingresos, a diferencia de Gómez Palacio, donde existen las tarifas normal y subsidiada; la primera se aplica en colonias de ingresos medios y altos, y la subsidiada en las de bajos. A los usuarios de Torreón que gastan entre uno y diez $\mathrm{m}^{3}$ se les cobra una tarifa base de 37.77 pesos, independientemente de su consumo, en tanto que en Gómez Palacio es de 38.51 y 22.2 pesos para la normal y subsidiada, respectivamente. Conforme el consumo aumenta, sube el precio. Al usuario que gasta $27 \mathrm{~m}^{3}$, se le cobrarán 4.44 pesos por cada uno y su facturación será de 119.88 pesos.

El consumo residencial de agua ha sido estudiado en muchos países durante las últimas décadas. Hansen (1996) analizó la demanda del líquido en Copenhague, Dinamarca, con un modelo que involucró variables como el precio del agua y la energía, el ingreso del consumidor y también las climáticas. El modelo se estimó usando series de tiempo, y se encontró una elasticidad precio cruzada de la energía de -0.2 y una del precio de la demanda de -0.1 .

Jones y Morris (1984) calcularon el consumo residencial de agua en Denver, Colorado, con dos formas para cuantificar el precio. La primera estimación se realizó con información completa de las cuotas y costos, y la otra con la formulación del precio promedio. El gasto anual de una familia se calculó con regresión en función de los pecios estimados, de su ingreso y tamaño; con una muestra de 326 observaciones, en 1976. Los resultados fueron adecuados al concepto especificado de precios, dada la variación proporcional en las cuotas y los costos, y consistentes con los encontrados en la literatura. Las elasticidades precios estimadas fueron -0.14 en el modelo lineal y -0.44 en el doble logarítmico, mientras que las elasticidades ingreso oscilaron entre 0.40 y 0.55 . 


\section{Cuadro 1}

Tarifa en pesos, para el agua de uso doméstico en la zona metropolitana de la Comarca Lagunera, enero de 2002

\begin{tabular}{|c|c|r|r|r|}
\hline \multicolumn{2}{|c|}{ Torreón } & \multicolumn{3}{|c|}{ Gómez Palacio } \\
\hline $\begin{array}{c}\text { Rango } \\
\text { metros } \\
\text { cúbicos }\end{array}$ & $\begin{array}{c}\text { Tarifa } \\
\text { normal }\end{array}$ & $\begin{array}{c}\text { Rango } \\
\text { metros } \\
\text { cúbicos }\end{array}$ & $\begin{array}{c}\text { Tarifa } \\
\text { normal }\end{array}$ & $\begin{array}{c}\text { Rango } \\
\text { subsidiada }\end{array}$ \\
\hline $0-10$ & 37.77 & $0-10$ & 38.51 & 22.20 \\
$11-20$ & 4.24 & $11-20$ & 3.78 & 2.22 \\
$21-30$ & 4.44 & $21-30$ & 3.83 & 2.26 \\
$31-40$ & 5.05 & $31-40$ & 4.00 & 2.35 \\
$41-60$ & 5.13 & $41-50$ & 4.09 & 2.40 \\
$61-100$ & 6.00 & $51-60$ & 4.20 & 2.45 \\
Más de 100 & 8.63 & $61-70$ & 4.56 & 2.60 \\
& & $71-80$ & 4.80 & 2.71 \\
& & $81-90$ & 5.08 & 2.84 \\
& & $91-100$ & 5.47 & 2.99 \\
& & $101-999$ & 6.86 & 3.78 \\
\hline
\end{tabular}

Fuente: elaborado con información obtenida de las gerencias comerciales de sIMAS en Torreón y de SIDEAPA en Gómez Palacio (2004).

El sistema de tarifas por bloques aplicado en Torreón contempla aumentos mensuales constantes durante el año; para 2002, 2003 y 2004, estos incrementos fueron de $0.55,0.42$ y 0.84 por ciento, respectivamente, y apenas lograron compensar la inflación. En Gómez Palacio las cuotas no han subido desde enero de 2002, lo que significa una disminución en términos reales.

Un consumo menor de agua se puede obtener por dos vías: la primera, con el aumento al cobro en el tiempo, en todos los rangos; así se lograría disminuir la demanda, en caso de que la cantidad consumida presentara alguna elasticidad a cambios en el precio. La segunda, con el incremento de la diferencia entre las tarifas de menor y mayor demanda; en este caso se lograría desincentivar el uso de quienes ubican su consumo en bloques mayores.

Debido a la importancia del agua en la Comarca Lagunera, el objetivo de la presente investigación es calcular elasticidades que midan la relación entre la cantidad del líquido demandada y las tarifas cobradas por los organismos operadores, a través del tiempo; para esto es necesaria la estimación de funciones de demanda de agua en el sector residencial de Torreón y Gómez Palacio. Puesto que el recurso es un bien necesario para la vida del ser huma- 
no, se espera que la cantidad requerida responda de manera inelástica a cambios en el precio.

\section{Metodología}

El modelo usado se basó en la teoría del consumidor, la cual establece que la cantidad demandada de un bien $\left(\mathrm{Q}_{\mathrm{d}}\right)$ depende de su precio $(\mathrm{P})$, del ingreso $(\mathrm{Y})$, del precio de los bienes sustitutos $\left(\mathrm{P}_{\mathrm{s}}\right)$, del precio de los bienes complementarios $\left(\mathrm{P}_{c}\right)$ y de otros factores como los gustos y preferencias, las expectativas de precios e ingresos y el número de consumidores (García et al. 2003, 23). La relación funcional entre la cantidad demandada del bien y los factores que la determinan se expone en los términos siguientes:

$$
\mathrm{Qd}=\mathrm{f}\left(\mathrm{P}, \mathrm{Y}, \mathrm{P}_{s}, \mathrm{P}_{c}, \mathrm{Z}\right)
$$

Donde $\mathrm{Z}$ incorpora al conjunto de elementos diferentes de $\mathrm{P}, \mathrm{Y}, \mathrm{P}_{s}, \mathrm{P}_{c}$. De acuerdo con la teoría económica, se espera una relación negativa entre $Q_{d} Y$ $P$. También, que $Q_{d}$ se relacione de manera positiva con $Y_{y} P_{s}$, si el bien analizado es normal y sustituto en el consumo o en forma negativa con $\mathrm{Y}_{\text {y }} \mathrm{P}_{c}$, si es inferior y complementario.

Con base en la teoría económica y el trabajo de Hansen (1996), se formuló una función de demanda doméstica de agua. En ella, la cantidad solicitada es la variable dependiente, en tanto que su precio, al igual que de la energía eléctrica, el ingreso, la precipitación pluvial y la temperatura son variables explicativas e independientes.

El modelo considera que el agua se usa en diferentes tareas del hogar, para la obtención de bienes finales. Entre ellas está la preparación de alimentos, higiene personal, limpieza de la casa, lavado de ropa y utensilios de cocina, entre otras. Cada tarea involucra el uso del recurso y, en la mayoría de los casos, de la energía eléctrica y otros bienes como aparatos electrodomésticos, jabón, etcétera.

Una función de demanda de agua puede proporcionar una estructura consistente, derivada de un modelo de producción doméstica de bienes de consumo final, que usa el líquido y la energía eléctrica. Si se asume que el gasto también está influido por el ingreso del usuario, y otras variables ambientales como la temperatura y la precipitación pluvial, la forma funcional por estimarse es la siguiente: 


$$
C A_{t}=\alpha_{0}+\alpha_{1} P A_{t-2}+\alpha_{2} Y_{t}+\alpha_{3} P E_{t}+\alpha_{4} P P_{t}+\alpha_{5} T E_{t}+e_{t}
$$

Donde $C A_{t}$ es la cantidad de agua requerida por vivienda en el mes t, $\mathrm{PA}_{\mathrm{t}-2}$ es el precio del bien en el mes $\mathrm{t}-2, \mathrm{PE}_{\mathrm{t}}$ es el precio de la energía eléctri$\mathrm{ca}$ en el mes $\mathrm{t}, \mathrm{Y}_{\mathrm{t}}$ es el ingreso en el mes $\mathrm{t}, \mathrm{PP}_{\mathrm{t}}$ es la precipitación pluvial en el mes $t, \mathrm{TE}_{\mathrm{t}}$ es la temperatura en el mes t y $e_{\mathrm{t}}$ es el término de error. La razón del rezago de dos meses en el precio del recurso es el pago bimestral por el servicio, una vez que el consumidor ha disfrutado de él.

La forma funcional, expresada en la función 2, es similar a la empleada por Hansen (1996) para estudiar la demanda del recurso, con la única diferencia de que él utiliza un índice agregado de variables ambientales para medir el comportamiento de la precipitación y la temperatura. En esta investigación, la función 2 se aplicó a usuarios de Torreón y Gómez Palacio (véase mapa), ciudades seleccionadas por su importancia económica y la escasez de agua, entre otras razones.

La estimación de la función de demanda se hizo con 84 observaciones, obtenidas de la combinación de datos de series de tiempo y sección cruzada; 28 de datos mensuales correspondientes al periodo de enero de 2002 a abril de 2004 en las colonias de ingresos bajos y las 56 restantes de valores mensuales —en el mismo lapso-, para las de percepciones medias y altas. Cada dato de cantidad demandada corresponde al valor promedio mensual observado en las colonias, de ingresos bajos, medios y altos, cuya selección se realizó por conveniencia, se eligieron cuidadosamente las más representativas según los ingresos bajos, medios y altos. Para Torreón se consideraron tres de bajos, dos de medios y tres de altos; en tanto que en Gómez Palacio una para cada uno.

La información sobre cantidad demandada por vivienda en diferentes colonias corresponde a gastos volumétricos medidos, y fue proporcionada en formato electrónico por personal del SIMAS de Torreón ${ }^{2}$ y del SIDEAPA de Gómez Palacio. ${ }^{3}$ Debido a que el sistema de tarifas de la Comarca Lagunera se caracteriza por ser de bloques crecientes, la considerada en cada observación correspondió al consumo. Conviene mencionar que los datos son de hogares cuyo medidor funciona, por consiguiente se descarta el informe de consumos fijos.

${ }^{2}$ Gerencia comercial de Torreón, junio de 2004.

3 Gerencia comercial de Gómez Palacio, junio de 2004. 
La Comisión Federal de Electricidad (CFE 2004) proporcionó la tarifa eléctrica. Se consideró la estacionalidad (temporadas de verano y fuera de verano) y rangos diferentes de consumo (menores y superiores a 175 y 225 $\mathrm{k} / \mathrm{W} / \mathrm{h}$ mensuales para fuera de verano y verano, respectivamente).

Los ingresos se calcularon conforme a los salarios mínimos que reciben las viviendas: bajos, medios y altos, según el Instituto Nacional de Estadística, Geografía e Informática (INEGI). Después, los salarios mínimos se multiplicaron por el mensual, para obtener el ingreso, éste corresponde al área geográfica $\mathrm{C}$, que incluye a Coahuila y Durango, que fue proporcionado por la Comisión Nacional de Salarios Mínimos (CONASAMI 2004).

En el caso de las variables ambientales, se tomaron en cuenta datos de temperatura y precipitación promedio mensual, obtenidos de la delegación estatal de la SAGARPA $(2003,14)$. Las variables monetarias fueron deflactadas con el índice de precios al consumidor de Torreón, obtenido del INEGI. Finalmente, la estimación del modelo se realizó con el paquete estadístico SAS.

\section{Resultados y discusión}

El análisis de los datos usados para calcular los modelos en las distintas colonias de Torreón y Gómez Palacio podría dar una idea sobre la demanda de agua en el sector residencial de la Comarca Lagunera (véase cuadro 2).

El consumo por vivienda está en función directa del ingreso, tal situación se observa en ambas ciudades. Entre menor sea éste, los hogares tienden a demandar menos agua y viceversa. El gasto mensual en las colonias de bajos ingresos de Torreón se concentró en los dos primeros rangos. Como se observa en el cuadro 2, 84.2 por ciento de las casas de ingresos bajos consumieron entre 1 y $20 \mathrm{~m}^{3}$, y requirieron 97.6 miles de $\mathrm{m}^{3}$, correspondientes a 69.6 por ciento del total. La demanda de agua en las colonias de ingresos altos se concentró en el tercer y cuarto rango; poco más de 30 por ciento de las viviendas consumieron entre 21 y $40 \mathrm{~m}^{3}$ al mes.

En las colonias de ingresos bajos, la demanda no excede los $100 \mathrm{~m}^{3} \mathrm{men}-$ suales. Las más altas se ubicaron en el rango de 81 a 90. Sin embargo, se detectaron 121 viviendas con requerimientos superiores a $100 \mathrm{~m}^{3}$ al mes y nueve hogares con un consumo mayor a 200.

En colonias de percepciones bajas de Gómez Palacio, 88.9 por ciento de la cantidad consumida se concentró en los primeros bloques, en tanto que el requerimiento en las de altas, 16.7 y 13.5 por ciento de las casas lo ubicaron en el tercer y cuarto bloque, respectivamente. 
A diferencia de las de Torreón, en las colonias de ingresos bajos de Gómez Palacio hay dos viviendas con gastos mayores a $100 \mathrm{~m}^{3}$. Y en las de ingresos altos, 26 y 4 hogares demandan más de 100 y $200 \mathrm{~m}^{3}$ al mes, respectivamente, durante el periodo estudiado (véase cuadro 2).

\section{Cuadro 2}

Cantidades mensuales de agua en metros cúbicos demandadas en Torreón y Gómez Palacio, abril de 2004

\begin{tabular}{|c|c|c|c|c|c|c|c|c|c|c|c|c|}
\hline \multirow{3}{*}{\begin{tabular}{|l|} 
Rango \\
Metros \\
cúbicos
\end{tabular}} & \multicolumn{4}{|c|}{ Ingresos bajos } & \multicolumn{3}{|c|}{ Ingresos medios } & \multicolumn{4}{|c|}{ Ingresos altos } & \\
\hline & \multicolumn{2}{|c|}{ Viviendas } & \multicolumn{2}{|c|}{$\begin{array}{c}\text { Consumo } \\
\text { de agua }\end{array}$} & \multicolumn{2}{|c|}{ Viviendas } & \multirow{2}{*}{$\begin{array}{l}\begin{array}{c}\text { Consumo } \\
\text { de agua }\end{array} \\
\begin{array}{c}\text { Metros } \\
\text { cúbicos }\end{array}\end{array}$} & \multicolumn{2}{|c|}{ Viviendas } & \multicolumn{3}{|c|}{$\begin{array}{l}\text { Consumo } \\
\text { de agua }\end{array}$} \\
\hline & Número & $\%$ & $\begin{array}{l}\text { Metros } \\
\text { cúbicos }\end{array}$ & $\%$ & Número & $\%$ & & $\%$ & Número & $\%$ & $\begin{array}{l}\text { Metros } \\
\text { cúbicos }\end{array}$ & $\%$ \\
\hline \multicolumn{13}{|l|}{ Torreón } \\
\hline $1-10$ & 2704 & 39.1 & 20346 & 20.8 & 115 & 12.7 & 837 & 3.3 & 204 & 8.1 & 1385 & 1.2 \\
\hline $11-20$ & 3115 & 45.1 & 47616 & 48.8 & 252 & 27.9 & 4141 & 16.2 & 334 & 13.2 & 5371 & 4.8 \\
\hline $21-30$ & 867 & 12.6 & 21470 & 22.0 & 241 & 26.7 & 6180 & 24.2 & 413 & 16.4 & 10737 & 9.5 \\
\hline $31-40$ & 182 & 2.6 & 6242 & 6.4 & 135 & 14.9 & 4791 & 18.8 & 428 & 17.0 & 15397 & 13.7 \\
\hline $41-50$ & 29 & 0.4 & 1296 & 1.3 & 86 & 9.5 & 3896 & 15.3 & 316 & 12.5 & 14416 & 12.8 \\
\hline $51-60$ & 6 & 0.1 & 344 & 0.4 & 30 & 3.3 & 1661 & 6.5 & 258 & 10.2 & 14396 & 12.8 \\
\hline $61-70$ & 1 & 0.0 & 62 & 0.1 & 14 & 1.5 & 908 & 3.6 & 187 & 7.4 & 12288 & 10.9 \\
\hline $71-80$ & 0 & 0.0 & 0 & 0.0 & 12 & 1.3 & 903 & 3.5 & 113 & 4.5 & 8524 & 7.6 \\
\hline $81-90$ & 3 & 0.0 & 247 & 0.3 & 9 & 1.0 & 768 & 3.0 & 88 & 3.5 & 7505 & 6.7 \\
\hline $91-100$ & 0 & 0.0 & 0 & 0.0 & 1 & 0.1 & 97 & 0.4 & 52 & 2.1 & 4967 & 4.4 \\
\hline $101-200$ & 0 & 0.0 & 0 & 0.0 & 9 & 1.0 & 1309 & 5.1 & 121 & 4.8 & 15475 & 13.8 \\
\hline Más 200 & 0 & 0.0 & 0 & 0.0 & 0 & 0.0 & 0 & 0.0 & 9 & 0.4 & 2024 & 1.8 \\
\hline Total & 6907 & 100.0 & 97623 & 100.0 & 904 & 100.0 & 25491 & 100.0 & 2523 & 100.0 & 112486 & 100.0 \\
\hline \multicolumn{13}{|c|}{ Gómez Palacio } \\
\hline $1-10$ & 307 & 49.6 & 2645 & 30.0 & 89 & 34.6 & 637 & 13.0 & 118 & 17.4 & 725 & 3.1 \\
\hline $11-20$ & 243 & 39.3 & 3599 & 40.8 & 88 & 34.2 & 1386 & 28.2 & 128 & 18.9 & 2008 & 8.5 \\
\hline $21-30$ & 50 & 8.1 & 1234 & 14.0 & 42 & 16.3 & 1038 & 21.1 & 153 & 22.6 & 3947 & 16.7 \\
\hline $31-40$ & 7 & 1.1 & 239 & 2.7 & 20 & 7.8 & 694 & 14.1 & 93 & 13.7 & 3249 & 13.8 \\
\hline $41-50$ & 4 & 0.6 & 184 & 2.1 & 11 & 4.3 & 521 & 10.6 & 62 & 9.1 & 2796 & 11.9 \\
\hline $51-60$ & 0 & 0.0 & 0 & 0.0 & 2 & 0.8 & 106 & 2.2 & 30 & 4.4 & 1635 & 6.9 \\
\hline $61-70$ & 1 & 0.2 & 64 & 0.7 & 2 & 0.8 & 131 & 2.7 & 25 & 3.7 & 1641 & 7.0 \\
\hline $71-80$ & 0 & 0.0 & 0 & 0.0 & 1 & 0.4 & 71 & 1.4 & 22 & 3.2 & 1645 & 7.0 \\
\hline $81-90$ & 2 & 0.3 & 164 & 1.9 & 0 & 0.0 & 0 & 0.0 & 11 & 1.6 & 942 & 4.0 \\
\hline $91-100$ & 3 & 0.5 & 290 & 3.3 & 1 & 0.4 & 95 & 1.9 & 6 & 0.9 & 590 & 2.5 \\
\hline $101-200$ & 1 & 0.2 & 174 & 2.0 & 0 & 0.0 & 0 & 0.0 & 26 & 3.8 & 3275 & 13.9 \\
\hline Más 200 & 1 & 0.2 & 226 & 2.6 & 1 & 0.4 & 232 & 4.7 & 4 & 0.6 & 1120 & 4.8 \\
\hline Total & 619 & 100.0 & 8819 & 100.0 & 257 & 100.0 & 4911 & 100.0 & 678 & 100.0 & 23573 & 100.0 \\
\hline
\end{tabular}

Fuente: elaborado con información obtenida de las gerencias comerciales de SIMAS en Torreón y de SIDEAPA en Gómez Palacio (2004).

Algunos indicadores estadísticos podrían dar una idea más cercana sobre el comportamiento del usuario de la zona metropolitana. En el cuadro 3 se muestran varios, como los valores mínimos y máximos, la media y la moda de las variables utilizadas en la estimación del modelo. 
En todas las colonias, el valor mínimo demandado fue de $1 \mathrm{~m}^{3}$ y el máximo osciló entre 83.1 y $393.0 \mathrm{~m}^{3}$ por mes. En Torreón, el valor máximo fue de 83.1, 271.8 y $246.0 \mathrm{~m}^{3}$ mensuales en las colonias de ingresos bajos, medios y altos, respectivamente; esto contrasta con los valores máximos en Gómez Palacio, en donde las cantidades fueron de 226.0, 232.0 y $393.0 \mathrm{~m}^{3}$. Hay colonias de ingresos bajos en Gómez Palacio, donde existen hogares con demandas muy elevadas, lo cual podría reflejar subsidios o bien fugas en el sistema de distribución.

El consumo promedio difiere en las dos ciudades, en Torreón el requerimiento de las colonias de ingresos bajos, medios y altos se ubicó en el segundo, tercer y cuarto rango, con $14.1,28.2$ y $44.6 \mathrm{~m}^{3}$, respectivamente. Tales resultados indican que la cantidad de agua demandada en las colonias de ingresos altos es 3.2 veces mayor, lo cual lleva a pensar que es posible una disminución. En Gómez Palacio, la demanda de las colonias de ingresos bajos y medios se ubicó en el segundo rango con 14.2 y $19.1 \mathrm{~m}^{3}$ de agua por mes, y la de altos fue de 34.8. En este caso, la cantidad solicitada por las colonias de ingresos altos representó 2.5 veces el consumo promedio de las de bajos.

El valor más frecuente de la muestra, la moda, fue de $10 \mathrm{~m}^{3}$, para las colonias de bajos ingresos en las dos ciudades; lo que permite concluir que sus consumidores tratan de pagar la tarifa menor, al ubicar su cantidad demandada en el límite del primer bloque. La moda en colonias de ingresos medios y altos de Torreón fue de 16 y $38 \mathrm{~m}^{3}$.

\section{Cuadro 3}

Indicadores estadísticos en metros cúbicos de consumo de agua en la Comarca Lagunera

\begin{tabular}{|l|r|r|r|r|c|}
\hline Estrato & $\begin{array}{c}\text { Tamaño } \\
\text { muestra }\end{array}$ & $\begin{array}{c}\text { Valor } \\
\text { mínimo }\end{array}$ & $\begin{array}{c}\text { Valor } \\
\text { máximo }\end{array}$ & Media & Moda \\
\hline Torreón & & & & & \\
\hline Ingresos bajos & 6907 & 1.0 & 83.1 & 14.1 & 10.0 \\
Ingresos medios & 904 & 1.0 & 271.8 & 28.2 & 16.0 \\
Ingresos altos & 2523 & 1.0 & 246.0 & 44.6 & 38.0 \\
\hline Gómez Palacio & 619 & 1.0 & 226.0 & 14.2 & 10.0 \\
\hline Ingresos bajos & 257 & 1.0 & 232.0 & 19.1 & 10.0 \\
Ingresos medios & 678 & 1.0 & 393.0 & 34.8 & 10.0 \\
Ingresos altos & & & & & \\
\hline
\end{tabular}

Fuente: elaborado con información obtenida de las gerencias comerciales de simas en Torreón y de SIDEAPA en Gómez Palacio (2004). 
En Gómez Palacio, la moda es de $10 \mathrm{~m}^{3}$ en las colonias de ingresos bajos, medios y altos. Este resultado es consecuencia de una administración ineficiente en el sistema de cobro, donde el consumidor paga la tarifa mínima, al reportar un consumo de diez $\mathrm{m}^{3}$ por mes.

En el cuadro 4 se muestran los resultados del estudio de regresión a partir de la estimación del modelo. En las ecuaciones 3 y 4 siguientes, se presentan los modelos calculados para Torreón y Gómez Palacio, respectivamente.

$$
\begin{aligned}
& \left.\overline{C A}_{t}=17.00-1.14 P A_{t-2}+0.0026 Y_{t}-24.39 P E_{t}+0.116 P P_{t}+0.24 T E_{t t} 3\right) \\
& \left.\overline{C A}_{t}=14.069-1.284 P A_{t-2}+0.00227 Y_{t}-15.72 P E_{t}+0.0779 P P_{t}+0.0172 T E_{t t} 4\right)
\end{aligned}
$$

El análisis estadístico de los resultados se realizó con base en Gujarati (1997, 72 y 122). Los coeficientes de determinación $\left(R^{2}\right)$ fueron 0.94 para las dos funciones. Las F calculadas (FC) fueron mayores a las F de tabla (Ft) $(\mathrm{p} \leq 10)$. Con la prueba de $\mathrm{t}$ se encontró que excepto la temperatura de la función de demanda de Gómez Palacio, todas las variables incluidas en el modelo fueron significativas. Con el valor de cada una de las variables independientes se obtuvo el valor predicho de la cantidad de agua demandada en Torreón y Gómez Palacio, que se alejó en 1.3 y 1.0 por ciento del valor promedio observado, respectivamente, lo cual indica que las variables consideradas en el modelo explican de manera satisfactoria el comportamiento de la cantidad de agua consumida.

En el análisis económico se juzgan los resultados obtenidos de la estimación de acuerdo a su conveniencia en el marco de la teoría económica, y se compara el signo de los parámetros estimados con algunos de sus principios. Si se analizan todas las ecuaciones, es posible apreciar que los coeficientes presentan el signo esperado.

Con los parámetros estimados y los valores medios de cada variable se calcularon las elasticidades del cuadro 4 . El valor de la elasticidad precio de la demanda indica que el agua responde de manera inelástica a cambios en la tarifa, tal resultado comprueba la hipótesis planteada inicialmente. La elasticidad resultó ser - 0.20 para Torreón y -0.18 para Gómez Palacio. Lo anterior significa que ante un aumento de 10 por ciento en el precio, la cantidad requerida disminuiría en 2 por ciento en Torreón y en 1.8 en Gómez Palacio. Estos valores indican que son necesarios los aumentos considerables en las tarifas actuales, para inducir al consumidor a disminuir la demanda. La baja elasticidad obedece a que se trata de un bien necesario para la vida del ser humano. Aunque las tarifas sufrieran aumentos considerables, la can- 
tidad demandada no sufriría cambios significativos, debido a la necesidad de seguir consumiendo el vital líquido. Tales cantidades coinciden con las cifras encontradas en otros trabajos.

El resultado anterior apoya el criterio de los organismos operadores, en relación con los aumentos mensuales en las tarifas, en Torreón éstos apenas igualan el crecimiento de la inflación y en Gómez Palacio no existen.

\section{Cuadro 4}

Resultados estadísticos, coeficientes estimados y elasticidades calculadas, enero de 2002 a abril de 2004

\begin{tabular}{|c|c|c|c|c|c|c|c|c|}
\hline \multirow{2}{*}{\begin{tabular}{|l|}
$\begin{array}{l}\text { Variable } \\
\text { dependiente }\end{array}$ \\
$C A_{\mathrm{t}}$ \\
\end{tabular}} & \multicolumn{2}{|c|}{ Intercepto } & \multicolumn{4}{|c|}{ Variables exógenas } & \multirow[t]{2}{*}{$\mathrm{R}^{2}$} & \multirow[t]{2}{*}{ Prob $>$ F } \\
\hline & & $P A_{t-2}$ & $Y_{t}$ & $\mathrm{PE}_{\mathrm{t}}$ & $\mathrm{PP}_{\mathrm{t}}$ & $T E_{t}$ & & \\
\hline \multicolumn{9}{|l|}{ Torreón } \\
\hline Coeficiente & 17.00 & -1.14 & 0.0026 & -24.39 & 0.116 & 0.24 & 0.94 & 0.0001 \\
\hline Error estándar & 12.36 & 0.97 & 0.00013 & 18.21 & 0.038 & 0.1549 & & \\
\hline Razón de t & 1.375 & -1.17 & 19.778 & -1.34 & 3.087 & 1.561 & & \\
\hline Elasticidad & & -0.20 & 0.94 & -0.48 & 0.08 & 0.19 & & \\
\hline \multicolumn{9}{|l|}{ Gómez Palacio } \\
\hline Coeficiente & 14.069 & -1.284 & 0.00227 & -15.72 & 0.0779 & 0.0172 & 0.94 & 0.0001 \\
\hline Error estándar & 4.267 & 0.60787 & 0.0002 & 6.44 & 0.03231 & 0.10556 & & \\
\hline Razón de t & 3.297 & -2.112 & 11.162 & -2.44 & 2.412 & 0.163 & & \\
\hline Elasticidad & & -0.18 & 0.98 & -0.43 & 0.05 & 0.02 & & \\
\hline
\end{tabular}

Fuente: elaborado con información obtenida del modelo estimado.

El costo de suministro del líquido en la Comarca Lagunera es inferior al precio cobrado por los organismos operadores. Datos de la CNA indican que en 2001 el costo fue de 1.6 pesos por $\mathrm{m}^{3}$ (CNA 2002, 62); éste que incluye el pago de la energía eléctrica y el mantenimiento de los equipos de bombeo, fue inferior a las tarifas de ese año, que superaron los tres pesos por $\mathrm{m}^{3}$. Los datos indican que no existen subsidios en el sector residencial, y esto aunado a la inelasticidad característica de la demanda significa que las alzas en las tarifas mayores a la tasa de inflación serían injustificadas.

De acuerdo con el valor de la elasticidad ingreso de la demanda, el agua se puede clasificar como un bien necesario; las elasticidades fueron 0.94 para Torreón y 0.98 para Gómez Palacio. Cuando el sueldo del usuario es mayor, le permite adquirir más y mejores satisfactores: aparatos electrodo- 
mésticos, casas más grandes con jardines amplios y alberca, etcétera, lo que en consecuencia aumenta el consumo de agua.

El valor de la elasticidad precio cruzada de la demanda indica que la energía eléctrica es un bien complementario del agua, en el proceso de producción de los hogares; dicha elasticidad resultó ser - 0.48 para Torreón y -0.43 para Gómez Palacio. Aumentos de 10 por ciento en la tarifa de la electricidad bajarán la demanda de este insumo, y disminuirán el gasto del líquido en 4.8 y 4.3 por ciento en Torreón y Gómez Palacio. Tal resultado se debe a que la energía eléctrica y el agua se usan de manera conjunta en procesos de producción del hogar, como la cocción y transformación de alimentos, el lavado y secado de ropa y el enfriamiento del ambiente en meses de verano.

Los subsidios a las tarifas de electricidad y el robo de ésta podrían estar estimulando el consumo de agua. Ante esta situación, la CFE, encargada de suministrar de energía eléctrica a la región, podría ayudar a hacer un uso más racional del recurso, mediante tarifas que reflejen el verdadero costo de producción y evitar la evasión del pago.

Las condiciones climáticas también explican los requerimientos de agua en la Comarca Lagunera, la elasticidad que relaciona la precipitación pluvial y la demanda del líquido resultó ser 0.08 para Torreón y 0.05 para Gómez Palacio. El valor positivo se debe a que la zona urbana no cuenta con drenaje pluvial que desahogue las precipitaciones, lo que ocasiona encharcamientos en banquetas y calles por varios días y suciedad que se limpia con agua potable. Además, la lluvia también aumenta la presión en las tuberías por varios días, y esto eleva el gasto en actividades como el baño personal diario.

La temperatura promedio es otra variable ambiental que incrementa el consumo de agua en Torreón. La elasticidad que relaciona la cantidad demandada y la temperatura promedio anual resultó ser 0.19 para Torreón y 0.02 para Gómez Palacio, la temperatura no mostró significancia.

\section{Conclusiones}

El consumo residencial de agua en la Comarca Lagunera mostró una respuesta inelástica con respecto a las tarifas pagadas. Por tanto, para lograr una disminución en el gasto se requieren cambios más que proporcionales en el precio pagado por el recurso. En la actualidad, las cuotas cobradas son mayores que el costo de extracción, por lo que no es recomendable un aumento de precios. Los incrementos nominales mensuales a las cuotas deberán igualarse a los de la inflación, para evitar que las tarifas disminuyan en términos reales. 
Si se quisiera reducir el gasto de agua en la región, se tendrían que subir las tarifas eléctricas, debido a que el consumo resultó ser más sensible a cambios en dicha variable.

Recibido en mayo de 2007 Revisado en septiembre de 2007

\section{Bibliografía}

Comisión Federal de Electricidad. 2004. Información al cliente. Conoce tu tarifa. http://www.cfe.gob.mx/es/InformacionAlCliente/conocetutari$\mathrm{fa} /$ (17 de agosto de 2004).

Comisión Nacional del Agua. 2002. Valor, costo y precio del agua en la región lagunera. México: CNA.

1997. Estudio del manejo de la demanda para la estabilización y recuperación de los acuíferos en la Comarca Lagunera, La Laja, Guanajuato y Caborca, Sonora. Región lagunera, Coahuila y Durango. Contrato GAS-11-PRO97. México: Ariel Consultores.

Comisión Nacional de Salarios Mínimos. 2004. Salarios mínimos generales y profesionales. http://www.conasami.gob.mx/ (27 de agosto de 2004).

García Mata, Roberto, J. Alberto García Salazar y Roberto C. García Sánchez. 2003. Teoría del mercado de productos agrícolas. Montecillo: Colegio de Postgraduados.

Gujarati, Damodar N. 1997. Econometría. Santa Fe de Bogotá: McGraw-Hill Interamericana.

Hansen, Lars Gårn. 1996. Water and Energy Price Impacts on Residential Water Demand in Copenhagen. Land Economics 72 (1): 66-79.

Jones, C. Vaughan y John R. Morris. 1984. Instrumental Price Estimates and Residential Water Demand. Water Resources Research 20 (2): 197-2002.

Secretaría de Agricultura, Ganadería, Desarrollo Rural, Pesca y Alimentación. 2003. Anuario estadístico de la producción agropecuaria en la Comarca Lagunera. Lerdo: SAGARPA, delegación regional. 